\title{
https://doi.org/10.46813/2021-132-029 BIAS OF BASAL DISLOCATION LOOP IN ZIRCONIUM
}

\author{
A.V. Babich, P.N. Ostapchuk \\ Institute of Electrophysics and Radiation Technologies NAS of Ukraine, \\ Kharkiv, Ukraine \\ E-mail:avbabich@kipt.kharkov.ua
}

An analytical expression for the elastic interaction energy of radiation point defects of the dipole type with the basal dislocation loop of the hcp metal is obtained using the Green's function method for hexagonal crystals in the Krener approach. It was used for numerical calculation of the bias for the basal dislocation loop of zirconium in a toroidal reservoir. The toroidal geometry of the reservoir allows one to perform the calculation for a loop of any size and without any correction of the elastic field in its region of influence. The dependencies of the loop bias on its radius and nature are obtained for various shapes of dipole defects.

PACS: 62.20.Dc; 62.20.Fe

\section{INTRODUCTION}

The concept of bias (preferential absorption of radiation point defects $(\mathrm{PD})$ of a certain type: vacancies or self-interstitial atoms (SIA), by internal extended crystal defects - sinks) is considered to be a central element of the theory of radiation-induced deformation. Such a feature of sinks (pores, dislocation loops and dislocation networks) determines the asymmetry of PD fluxes on them and leads to the evolution of the material microstructure and its macroscopic deformation. Typical examples of such deformation are the radiation swelling of metals with cubic symmetry [1] and radiation growth $(\mathrm{RG})$ of hcp metals and, in particular, zirconium - the main structural material of the cladding of fuel elements of nuclear reactors of the WWER type [2]. According to classic elastic ideology (EID - elastic interaction difference [3]), the concept of the dislocation bias is based on a stronger elastic interaction of interstitial atoms with the edge components of dislocations as compared to vacancies. It is generally accepted that dislocations preferentially absorb SIAs, while vacancies remaining in excess are absorbed by other sinks, causing their diffusion evolution. This is how the phenomena of vacancy swelling of cubic metals and alloys are explained [4-6]. However, attempts to apply the ideas of EID to explain the RG phenomenon of hcp metals have not yet reached their goals. The RG is accompanied by a change in the shape of the material without the application of an external load and without a noticeable change in volume. Thus zirconium expands in the $\langle\mathrm{a}\rangle$-direction and shrinks along the $\langle\mathrm{c}\rangle$-axis during the process of radiation growth $[7,8]$. This is possible if, for example, on the basal planes of zirconium, vacancy loops are nucleated and grow, "eating up" the crystal along $<\mathrm{c}>$-axis. On the prismatic ones - the process of nucleation and growth of interstitial loops takes place, which leads to forming additional extraplanes in the <a>-direction [2] (Fig. 1). However, the mechanisms of nucleation and growth of vacancy loops are not clear yet, since, according to the EID [9], the bias factor of the loop does not depend on loop nature. One should note that there is an alternative version of the EID theory in the literature. This is the ideology of PD anisotropic diffusion (DAD diffusional anisotropy difference [8, 10]). Its main assumption is as follows $D_{i}^{a} / D_{i}^{c}>D_{v}^{a} / D_{v}^{c}$. Here $D_{m}^{a}$ is the diffusion coefficient of PD of $m$-type in the basal plane of zirconium, $D_{m}^{c}$ is the coefficient of diffusion in $\langle c\rangle$-direction (subscript $i$ and $v$ refer to vacancies and SIA respectively). Then it turns out that straight edge dislocations in the basal and prismatic planes move in opposite directions, i.e., vacancies migrate predominantly to the basal zirconium plane, and interstices move to the prismatic one. Thus, there is a potential possibility of the growth of basic vacancy loops. However, there is no experimental confirmation of this inequality to date. Moreover, recent numerical calculations [11] have shown that vacancies and SIA really migrate diffusionally mainly parallel to the basal plane $\left(D_{i, v}^{a} / D_{i, v}^{c}>1\right)$, however, the inequality in the range of reactor temperatures $(T<800 \mathrm{~K})$ is just the opposite $D_{i}^{a} / D_{i}^{c}<D_{v}^{a} / D_{v}^{c}$. Therefore, the authors of [11] question the possibility of explaining the observed growth of zirconium by the theory of anisotropic diffusion DAD, which means that the theory of the elastic "bias" remains relevant. In this regard, an analytical expression for the elastic interaction energy of radiation point defects of the dipole type with the basal dislocation loop of the hcp metal was obtained using the Green's function method for hexagonal crystals in the Krener approach. The bias for the basic edge loop of zirconium of various types was numerically calculated. The possibility of further application of elastic ideology to explain the RG of zirconium is discussed. 


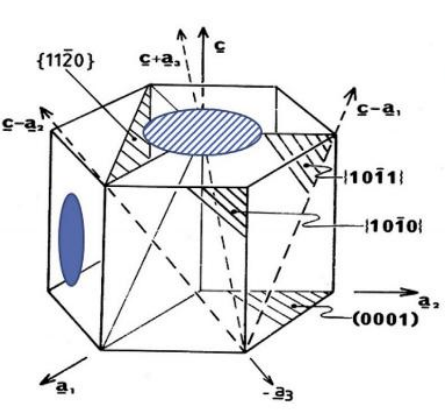

Slip Planes and Dislocation Burgers Vectors

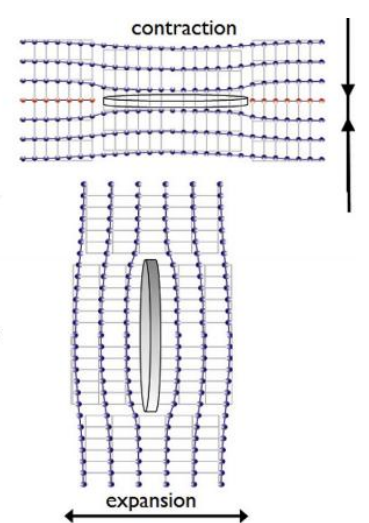

Fig. 1. Schematic showing irradiation growth in Zr. Interstitial loops form on (1010) planes and vacancy loops form on basal (0001) planes [2]

\section{ELASTIC INTERACTION ENERGY}

If the fictitious distribution of volume forces $f_{i}^{S}$ that creates the same stresses in an elastic medium as a real source $S$ are known, then the interaction energy between two systems of internal stresses $S\left(\boldsymbol{u}^{S}, u_{i j}{ }^{S}, \sigma_{i j}{ }^{S}\right)$ and $T\left(\boldsymbol{u}^{T}\right.$, $\left.u_{i j}{ }^{T}, \sigma_{i j}{ }^{T}\right)$, according to Eshelby [12], can be represented by an integral of the form:

$$
E_{\text {int }}(S, T)=-\int f_{i}^{S} u_{i}^{T} d V
$$

which is calculated over the area containing only the source of the system $S$. Let the source $S$ be a PD of the dipole type described in elasticity theory by the volume distribution of dipole forces without a moment

$$
f_{i}^{S}(\boldsymbol{r})=-P_{i j} \nabla_{j} \delta(\boldsymbol{r}-\boldsymbol{a}), P_{i j}=P_{j i},
$$

in addition, a source of inner stresses $T$ is a dislocation loop $D$. Then the interaction energy takes the following form:

$$
E_{\text {int }}(\boldsymbol{r})=-\int f_{i}\left(\boldsymbol{r}^{\prime}\right) u_{i}^{D}\left(\boldsymbol{r}^{\prime}\right) d \boldsymbol{r}^{\prime}=-P_{i j} u_{i j}^{D}(\boldsymbol{r}) .
$$

Here $u_{i j}{ }^{D}(\boldsymbol{r})$ - deformation field caused by the loop in the point $r=a$ where the PD is situated. If the elastic dipole has an axis of symmetry which coincides with $<\mathrm{c}>$-axis of hpc crystal, then tensor $P_{i j}$ has only diagonal components, which in the abbreviated description can be written as $P_{i}=P(1,1, \varepsilon), P=P_{a}, \varepsilon=P_{c} / P_{a}$. Here $P_{c}$ and $P_{a}$ - strength of the force-dipoles in $\langle a\rangle$ - and $\langle c\rangle$ directions. Note that $P_{a}$ and $P_{c}$ do not have a simple physical meaning for defect, which is described by force density (2), in crystal with non-cubic symmetry. Therefore, in papers $[13,14]$, by analogy with $P_{i}=P(1,1, \varepsilon)$, displacement dipoles $Q_{i}=Q(1,1, \delta), Q=Q_{a}$, $\delta=Q_{c} / Q_{a}$ were introduced. They are connected with force-dipoles by relation $P_{i}=C_{i j} Q_{j}$. Here $C_{i j}$ is the crystal elastic moduli. It was assumed that the change in the volume $\Delta V$ of the finite crystal caused by a point defect is related to the displacement dipoles by the relation $\Delta V=Q(2+\delta)$. Then for hexagonal crystal:

$$
\begin{gathered}
E_{\text {int }}(\boldsymbol{r})=-\Delta V C\left[u_{11}^{D}(\boldsymbol{r})+u_{22}^{D}(\boldsymbol{r})+\varepsilon u_{33}^{D}(\boldsymbol{r})\right], \\
C=\frac{C_{11}+C_{12}+\delta(\varepsilon) C_{13}}{2+\delta(\varepsilon)}, \\
\delta(\varepsilon)=\frac{\varepsilon\left(C_{11}+C_{12}\right)-2 C_{13}}{C_{33}-\varepsilon C_{13}} .
\end{gathered}
$$

In case of dilatation center $(\varepsilon=1)$ and elastically isotropic medium $\left(C_{11}=C_{22}=C_{33}=\lambda+2 \mu\right.$; $C_{12}=C_{13}=\lambda$ ) one can get:

$$
E_{\text {int }}(\boldsymbol{r})=-\Delta V K S p u_{i j}^{D}(\boldsymbol{r}) .
$$

Here $K$ is the bulk modulus. Thus, it remains to calculate the deformation field created by a particular loop in a particular elastic medium.

\section{ELASTIC STRAIN FIELD OF THE BASAL DISLOCATION LOOP IN A HEXAGONAL CRYSTAL}

Consider an edge vacancy loop of radius $\mathrm{R}$ lying in the plane $z=0$ (zirconium basal plane) of a cylindrical system coordinates $(r, \varphi, z)$, the Burgers vector of which is perpendicular to the loop plane and has only the $\mathrm{z}$ component $\left(0,0, b^{D}\right)$ (Fig. 2). The normal vector to the plane of the loop coincides with the positive direction of the z-axis, which is also the symmetry axis of the crystal.

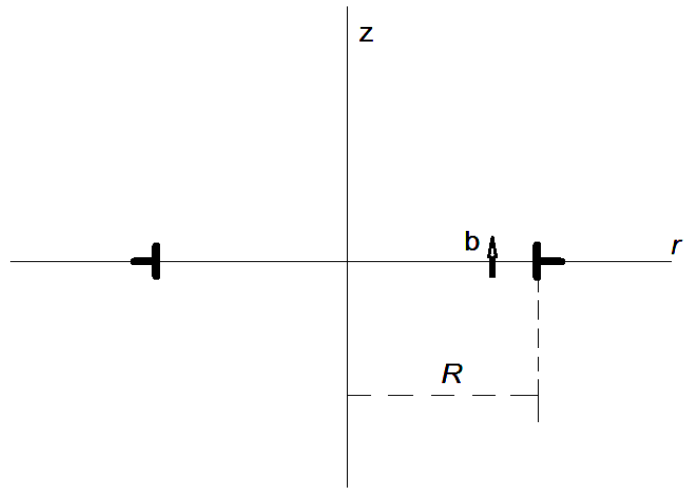

Fig. 2. A vacancy loop of radius $R$ lying in the plane $z=0$ of the cylindrical coordinate system in $(r, \varphi, z)$

with the Burgers vector $\boldsymbol{b}$ along the " $z$ "- axis

Let us recall that the formalism of the tensor Green's function $G_{i j}$ of the equations of equilibrium of an elastic medium allows one to calculate the displacements created by a dislocation of any shape in any anisotropic elastic medium, according to the classical formula [15]:

$$
u_{i}^{D}(\boldsymbol{r})=C_{j k l m} b_{m}^{D} \int_{S_{D}} n_{l}^{D} \frac{\partial G_{i j}\left(\boldsymbol{r}-\boldsymbol{r}^{\prime}\right)}{\partial x_{k}} d S^{\prime} .
$$

Here $C_{j k l m}$ is the tensor of elastic moduli of the medium, modeling crystal; $b_{m}{ }^{D}$ is the $m$ - component of the dislocation Burgers vector; $n_{l}^{D}$ is $l$-component of the normal vector to an arbitrary surface $S_{D}$, based on the dislocation line; $\boldsymbol{r}$ is the observation point coordinate; $\boldsymbol{r}$ ' is the surface point coordinate $S_{D}$. In paper [16], using Kröner approach [13] and taking into account corrections [14], the following analytical expressions were obtained for interaction energy (4) and displacements (5)

$$
\begin{gathered}
u_{11}^{D}(\boldsymbol{r})+u_{22}^{D}(\boldsymbol{r})=\frac{b^{D}}{4 \pi} \int_{S_{D}} \frac{d^{2} r^{\prime}}{\left|\boldsymbol{r}-\boldsymbol{r}^{\prime}\right|^{3}}\left[C_{13} \mathrm{~T}_{1}\left(\tau_{3}^{2}\right)+C_{33} \mathrm{~T}_{2}\left(\tau_{3}^{2}\right)\right] ;(6) \\
u_{33}^{D}(\boldsymbol{r})=\frac{b^{D}}{4 \pi}\left(C_{13}+C_{33}\right) \int_{S_{D}} \frac{d^{2} r^{\prime}}{\left|\boldsymbol{r}-\boldsymbol{r}^{\prime}\right|^{3}} \mathrm{~T}_{3}\left(\tau_{3}^{2}\right) \\
\mathrm{T}_{1}=\sum_{\alpha=1}^{2} A_{\alpha} v_{\alpha}^{2} F_{\alpha}\left(\tau_{3}^{2}\right) ; \quad \mathrm{T}_{2}=\sum_{\alpha=1}^{2} D_{\alpha} F_{\alpha}\left(\tau_{3}^{2}\right)
\end{gathered}
$$




$$
\begin{gathered}
\mathrm{T}_{3}=\sum_{\alpha=1}^{2} C_{\alpha} v_{\alpha} F_{\alpha}\left(\tau_{3}^{2}\right) ; \\
F_{\alpha}\left(\tau_{3}^{2}\right) \equiv \frac{2 \tau_{3}^{2}-v_{\alpha}\left(1-\tau_{3}^{2}\right)}{\left[\tau_{3}^{2}+v_{\alpha}\left(1-\tau_{3}^{2}\right)\right]^{5 / 2}} ; \quad \tau_{3}^{2}=z^{2} /\left|\boldsymbol{r}-\boldsymbol{r}^{\prime}\right|^{2} ; \\
A_{\alpha}=\left[\left(C_{66}-C_{11}\right)\left(C_{33}-v_{\alpha} C_{44}\right)+\left(C_{13}+C_{44}\right)^{2}\right] / E_{\alpha} ; \\
C_{\alpha}=\frac{\left(C_{13}+C_{44}\right)\left(C_{44}-v_{\alpha} C_{66}\right)}{E_{\alpha}} ; \quad C_{66}=\frac{\left(C_{11}-C_{12}\right)}{2} ; \\
D_{\alpha}=\left(C_{44}-v_{\alpha} C_{11}\right)\left(C_{44}-v_{\alpha} C_{66}\right) / E_{\alpha} ; \\
E_{1}=C_{11} C_{44} C_{66}\left(v_{1}-v_{3}\right)\left(v_{1}-v_{2}\right) ; \\
E_{2}=C_{11} C_{44} C_{66}\left(v_{2}-v_{3}\right)\left(v_{2}-v_{1}\right) .
\end{gathered}
$$

Here $v_{\alpha}$ are solutions of the equation:

$$
C_{44} C_{11} v^{2}+\left(C_{13}{ }^{2}+2 C_{44} C_{13}-C_{33} C_{11}\right) v+C_{44} C_{33}=0 .
$$

Hence the problem of calculation of the interaction energy between PD and base loop $\left(z^{\prime}=0\right)$ is reduced to integrating (6) over the loop area. All further calculations are performed using dimensionless coordinates $\quad r \rightarrow r / b, \quad z \rightarrow z / b$, $\left|\boldsymbol{r}-\boldsymbol{r}^{\prime}\right|^{2}=r^{2}+z^{2}-2 r r^{\prime} \cos \left(\varphi-\varphi^{\prime}\right)+r^{\prime 2}$. Due to isotropy in the crystal basal plane, the dependence on the azimuthal angle $\varphi$ in (6) is absent, so let us set $\varphi$ equal to zero. The distance dependence of the dimensionless $\left(E_{\text {in }} / k T\right)$ interaction energy of SIA with the vacancy loop of the radius $R=10 b$ in zirconium is shown in Fig. 3 for value of $z=5 b$ and different values of $\varepsilon$.

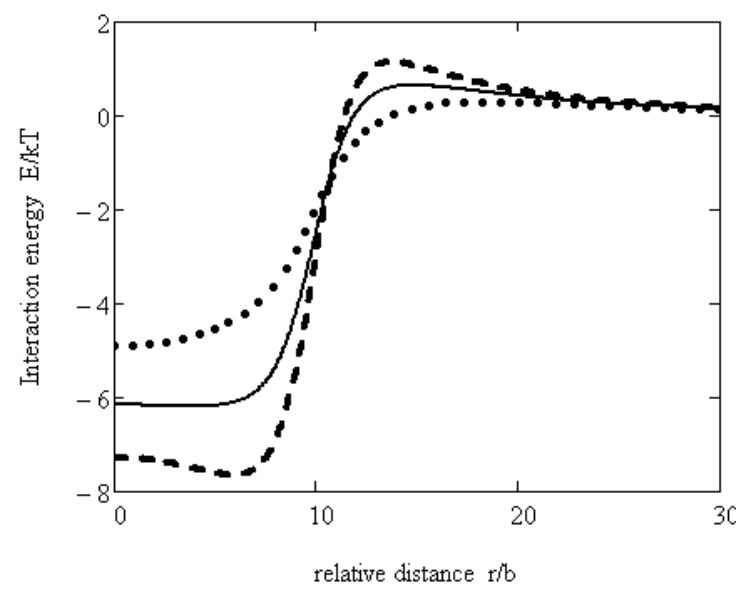

Fig. 3. Dependence of dimensionless $\left(E_{\text {in }} / k T\right)$ interaction energy of SIA with vacancy loop with the radius $R=10 \mathrm{~b}$ in zirconium on distance $\rho=r / b$ $(\varepsilon=0.8-$ point line; $\varepsilon=1.0-$ solid line; $\varepsilon=1.2-$ dotted line)

Experimental values of the elastic moduli of zirconium according to [17] (Mbar): $C_{11}=1.154$; $C_{12}=0.672 ; \quad C_{13}=0.646 ; \quad C_{33}=1.725$; $C_{55}=C_{44}=0.363$. Other values: $T=573 \mathrm{~K}, \Delta V=1.2 \omega$, $\omega=2.35^{*} 10^{-29} \mathrm{~m}^{3}$. One can see that an interstitial atom in the form of an elongated (along with $\langle c\rangle$-axis) ellipsoid of rotation $(\varepsilon=1.2)$ is attracted (the inner region of the loop) and repelled (outer) stronger than the center of dilatation $(\varepsilon=1)$ and especially stronger than the oblate spheroid $(\varepsilon=0.8)$. The vacancy interacts with the loop in a similar way, but with a correction for the sign $(\Delta V=-0.6 \omega)$.

\section{LOOP BIAS. FORMULATION OF THE PROBLEM}

As is known, the sink bias is determined by a relation of the form $B=1-Z_{v} / Z_{i}$, here subscripts $v$ and $i$ correspond to vacancies and SIA respectively. If $B>0$, one says that the loop has a preference to SIA. The dimensionless quantity $Z_{v, i}$ is called the absorption efficiency of the PD by the sink. It appears as a result of calculating the PD diffusion flux to a specific sink. In our case, under the assumption of diffusion isotropy of the medium $\left(D_{i j}=D \delta_{i j}\right)$ the PD flux $J$ to the dislocation loop is found by solving the following diffusion problem in its region of influence using the quasistationary approximation:

$$
\begin{aligned}
& \omega \operatorname{div} \boldsymbol{j}(\boldsymbol{r})=0 ; \omega \boldsymbol{j}(\boldsymbol{r})=-D C(\boldsymbol{r}) \beta \nabla \mu(\boldsymbol{r}) ; \\
& \beta \equiv 1 / k_{B} T ; \\
& \beta \mu(\boldsymbol{r})=\ln \left(\frac{C(\boldsymbol{r})}{C^{e}} \exp \left(\beta E_{\mathrm{int}}(\boldsymbol{r})\right)\right) ; \\
& J=\iint_{S}[\boldsymbol{n} \boldsymbol{j}(\boldsymbol{r})] d \sigma .
\end{aligned}
$$

Here $C(r)$ is the concentration of migrating PD; $\boldsymbol{j}(\boldsymbol{r})$, $\mu(r)$ - their flux density and chemical potential, respectively; $E_{\text {int }}(\boldsymbol{r})$ - their interaction energy (equations (4), (6)) with the loop; $C^{e}$ - equilibrium thermal concentration of PD in the crystal in the absence of a stress field $E_{\text {int. }}$. The integral is taken over an arbitrary surface containing the loop with the outer normal $\boldsymbol{n}$. Equation (7) should be supplemented with boundary conditions. The inner surface $S_{c}$ is usually chosen in the form of a torus containing a dislocation line. The torus minor radius $r_{c}$ corresponds to the dislocation core radius. The boundary condition on it has the form:

$$
\left.C(\boldsymbol{r}) \exp \left(E_{\text {int }}(\boldsymbol{r})\right)\right|_{S_{c}}=C_{R} .
$$

The condition has the traditional form and corresponds to the value of the PD chemical potential at the dislocation core $\left.\mu\right|_{S_{c}}=\frac{\omega}{b} P\left(r_{c}, R\right)$. Here $P$ is the force per unit length of the loop applied normally to the dislocation line in its plane. The force is determined by the linear tension of the dislocation line and determines the effect of coalescence of loops of the same nature upon annealing. Outer surface $S_{\text {ext }}$ can be selected in different forms: it is either a sphere, or a cylinder, or a torus coaxial with $S_{c}$ with generating circle radius $R_{e x t}$ which corresponds to the radius of the loop influence region. Usually the boundary condition on the $S_{e x t}$ is formulated [9] in the form $\left.C(\boldsymbol{r})\right|_{S_{e x t}}=\bar{C}$. Here $\bar{C}$ is the average PD concentration in an effective medium that simulates the influence of all sinks. We use the different form of the boundary condition. We formulate it for the chemical potential in the form: $\left.\beta \mu\right|_{S_{e x t}}=\ln \left(\bar{C} / C^{e}\right)$. This is the standard form of the PD chemical potential in an effective medium, where the influence of a particular sink is neutralized by the others. Then:

$$
\left.C(\boldsymbol{r}) \exp \left(E_{\mathrm{int}}(\boldsymbol{r})\right)\right|_{S_{e x t}}=\bar{C} .
$$

In terms of a variable 


$$
\psi(r, z)=\left[C(r, z) \exp E_{\text {int }}(r, z)-C_{R}\right] /\left[\bar{C}-C_{R}\right],
$$

the quasi-stationary diffusion equation in dimensionless cylindrical coordinates taking into account isotropy in the basal plane has the form:

$$
\frac{\partial^{2} \psi}{\partial r^{2}}+\frac{\partial^{2} \psi}{\partial z^{2}}+\left(\frac{1}{r}-\frac{\partial E_{\text {int }}}{\partial r}\right) \frac{\partial \psi}{\partial r}-\frac{\partial E_{\text {int }}}{\partial z} \frac{\partial \psi}{\partial z}=0
$$

with boundary conditions on the inner and outer toroidal surfaces:

$$
\begin{gathered}
\psi(r, z)=0 \quad \text { on } \quad\left(r^{2}+z^{2}+R^{2}-r_{c}^{2}\right)^{2}=4 R^{2} r^{2} ; \\
R-r_{c} \leq r \leq R+r_{c} ;
\end{gathered}
$$

$$
\psi(r, z)=1 \quad \text { on } \quad\left(r^{2}+z^{2}+R^{2}-R_{e x t}{ }^{2}\right)^{2}=4 R^{2} r^{2}
$$

$$
R-R_{\text {ext }} \leq r \leq R+R_{\text {ext }} \quad \text { for } \quad R>R_{\text {ext }} \text {; }
$$$$
0 \leq r \leq R+R_{\text {ext }} \quad \text { for } \quad R<R_{\text {ext }} \text {. }
$$

Then for the flux and absorption efficiency $\mathrm{Z}_{\mathrm{v}, \mathrm{i}}$ we have:

$$
\begin{gathered}
J=2 \pi R \frac{D}{\omega} Z\left(r_{c}, R, R_{e x t}\right)\left[\bar{C}-C_{R}\right], \\
Z\left(r_{c}, R, R_{e x t}\right)=\frac{1}{2 \pi R} \iint_{S} \mathrm{e}^{\left(-E_{\text {int }}(r, z)\right)}[\boldsymbol{n} \nabla \psi(r, z)] d \sigma .
\end{gathered}
$$

The diffusion problem (11), (12) was solved numerically by the finite difference method. Fig. 4 shows a cross section of a toroidal reservoir containing a loop, taking into account the reflection symmetry in the plane $z=0$ and symmetry about rotation around the z-axis. For radius $R>R_{\text {ext }}$, the diffusion field was calculated in the region bounded by the surfaces DA, $\mathrm{AB}, \mathrm{BC}, \mathrm{CD}$, for $R<R_{\text {ext }}$ by the surfaces $\mathrm{OA}, \mathrm{AB}, \mathrm{BC}$, $\mathrm{CD}$, DO. The specified symmetry imposes additional boundary conditions: $\partial \psi / \partial z=0$ on DA, BC, OA, corresponding to zero flux through the plane $z=0$, and $\partial \psi / \partial r=0$ on DO (axis of symmetry). After that, using (14), we calculated the absorption efficiency of the $\alpha$ type PD and the bias $B$. An arbitrary inner surface $S$ in (14) is chosen for the convenience of calculations in the form of a rectangle of rotation. In Fig. 4, this is the contour $L$. The calculations were performed for zirconium, the material parameters of which are given above. A point defect was modeled by a displacement dipole (see Fig. 3).
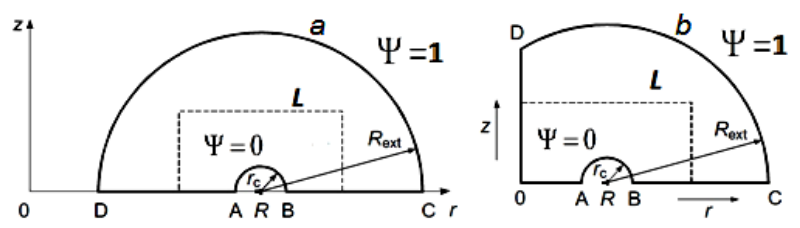

Fig. 4. Coordinate system for a toroidal reservoir: $a-R>R_{\text {ext }}, b-R<R_{\text {ext }}$

\section{RESULTS AND ITS DISCUSSION}

It should be noted that the Green's functions method was used here for a reason. The point is that the next step is the estimation of the prismatic loop bias. This method is the only one way of calculating the elastic interaction energy of a PD with a loop in a prismatic plane. For the basal loop, as is known, there is an alternative Elliott method [18], but there is no alternative for the prismatic one. The Green's function method is more complicated and cumbersome, so it was important to check whether the method [9] works in the case of basal loop.
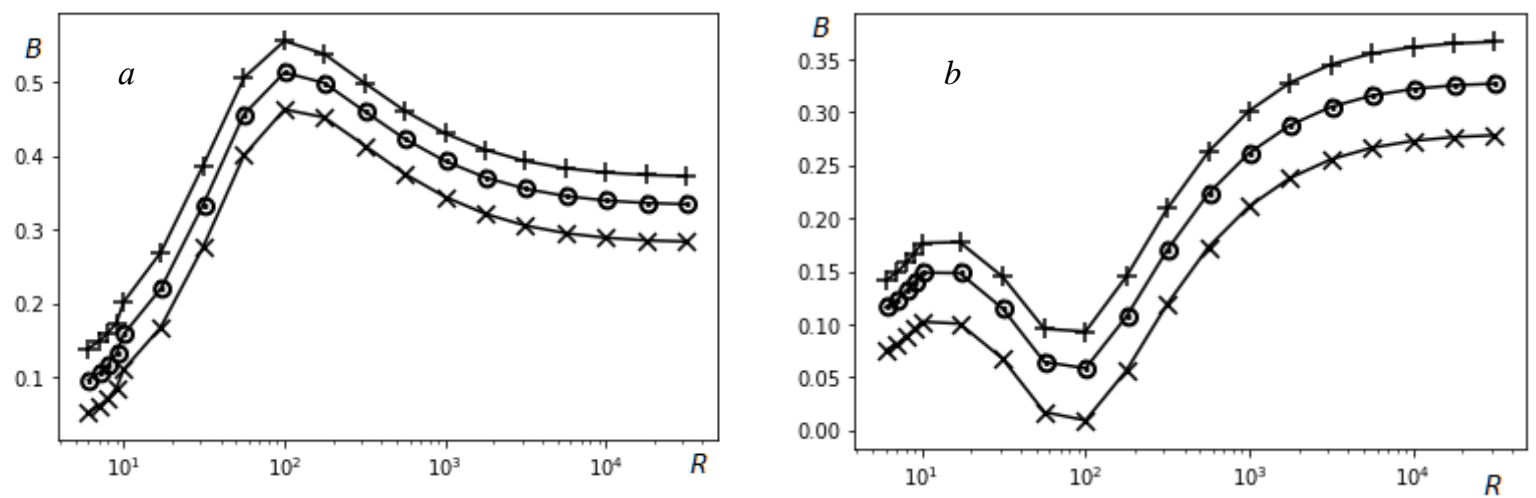

Fig. 5. The dependencies of the biases of vacancy and SIA dislocation loops on their radiuses in units of $b$ in 3 cases: $\varepsilon=0.8$ ('X'), $\varepsilon=1.0$ ('O'), and $\varepsilon=1.2(+)(a-$ vacancy loop, $b-$ SIA loop $)$

Fig. 5,a shows the dependence of the bias of the vacancy dislocation loop on its radius in units of $b$ in 3 cases: $\quad \varepsilon=0.8$ (' $\times$ ' - line), $\quad \varepsilon=1.0$ (' $\mathrm{O}$ ' - line), and $\varepsilon=1.2('+$ ' - line). The cases correspond to Fig. 3 and $R_{\text {ext }}=55 \mathrm{~b}$. To simplify calculations, the radius of the cross section of the outer torus $R_{\text {ext }}$ was set to be the same for vacancies and SIA. It corresponds to the approximation $\rho \approx 1 / \pi R_{e x t}^{2}$ (here $\rho$ is the sink density). If dislocations are the dominant sink in the system, then the value $R_{e x t}=55 b$ corresponds to the dislocation density $\rho \approx 10^{11} \mathrm{~cm}^{-2}$. Fig. 5,b shows the same dependencies for SIA loop. From Fig. 5 one can make conclusion that the shape of PD does not change qualitatively the dependence of the loop bias on loop radius.

There were many attempts of solving similar problems, but using the approximation of an elastically isotropic medium, as well as the cylindrical or the spherical reservoir. The main results of these studies are formulated in [9]. Actually, dislocation loops are biased sinks, which absorb SIAs more efficiently than vacancies, since $B>0$ for both types of loops (see Fig. 5). The bias depends on the loop radius and the sink density. However, in [9] there is an important conclusion that the bias does not depend on the nature 
of the loop independently on the type of reservoir. In our case, one can see the significant dereference between the absorption of PD by loops of different nature. The bias of the interstitial loop at a fixed outer radius of the torus $R_{e x t}$ has a minimum, reaching the corresponding values of the straight dislocation (limit $R \rightarrow \infty)$. By the way, the last feature is typical for a toroidal reservoir; in the case of a sphere or a cylinder, $B$ increases monotonically. A similar dependence for the vacancy loop demonstrates the presence of a maximum. Note that the bias for loops of different nature with an increase in their size goes to the same value, as it should be in theory. The formal reason for the mentioned above independence of the bias on the loop radius is associated with the boundary condition at the outer radius of the torus $R_{e x t}\left(\left.C(\boldsymbol{r})\right|_{S_{e x t}}=\bar{C}\right)$. In our approach, we use different boundary condition (9) which is not sensitive to loop type. As a result, we have one absorption efficiency $Z\left(r_{c}, R, R_{e x t}\right)$, the flux per loop proportional to the difference $\left[\bar{C}-C_{R}\right]$. Using of the boundary conditions (9) leads to appearance of two efficiencies: absorption and emission, respectively, and two fluxes and, as a result, to the independence of the absorption bias on the nature of the loop. Up to now, there is no proper answer to the question: which approach is correct? In our version, the basis interstitial loops with the smallest bias can be considered the main sinks for vacancies. Therefore, they have no chance of survival, which is observed in experiments. That fact is encouraging. The future of vacancy loops is ambiguous. Large loops cannot survive because of their larger bias compared to straight dislocations; however, these loops are still observed during the radiation growth of zirconium. Their "accumulation point" can be considered the loop size which corresponds to $B=B_{\text {str }}$ (Fig. 6).

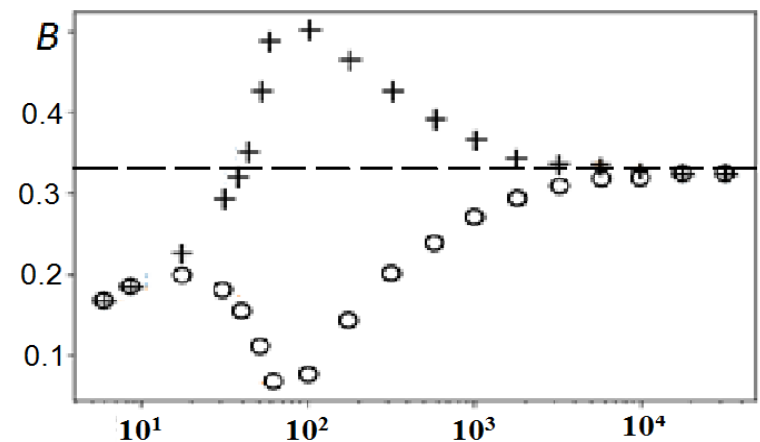

Fig. 6. Dependency of the loop bias on the loop radius for $R_{\text {ext }}=55 b$ ( “o" - interstitial loop,

$$
\text { “+”-vacancy loop) }
$$

If during the evolution of the crystal microstructure the average bias of the sink system as a whole becomes larger than bias of a straight dislocation and grows, then the "accumulation point" can shift towards an increase in the size of the surviving vacancy loops. In other words, some "external" sources of vacancies in the basal plane are required. Possibly, the interstitial loops, which arise on prismatic planes during the radiation growth of zirconium, can play the role of such sources.
Therefore, a similar analysis of their bias factor should be the subject of future research.

\section{CONCLUSIONS}

The Green's functions method used here for a reason. It is the only one that may be used for calculation of the interaction energy between radiation PDs and dislocation basal and prismatic loops in GPU metals. PDs was modeled as force-dipoles. Elliott's method may be used only for basal loops. The dependence of basal loop bias on loop radius in zirconium was calculated numerically with boundary conditions using chemical potential. It was shown that it leads to significant dereference between our results and standard results [9]. It was shown that the shape of PD does not change qualitatively the dependence of the loop bias on loop radius. We are going to apply the methodology used in this article to calculate PD fluxes and bias for prismatic loops in zirconium in order to solve the problem of its radiation growth.

This study was performed within the Ukrainian State program Support for the Development of Priority Fields of Scientific Research (KPVK 6541230).

\section{REFERENCES}

1. В.Н. Воеводин, И.М. Неклюдов. Эволюиия структурно-фазового состояния и радиационная стойкость конструкционных материалов. Киев: «Наукова думка», 2006, 376 с.

2. R.B. Adamson, C.E. Coleman, M. Griffiths // J. Nucl. Mater. 2019, v. 521, p. 167.

3. C.H. Woo // J. Nucl. Mater. 1988, v. 159, p. 237.

4. A.D. Brailsford, R. Bullougb // J. Nucl. Mater. 1972, v. 44, p. 121.

5. P.T. Heald, M.V. Speight // Acta Met. 1975, v. 23, p. 1389.

6. A.D. Brailsford, R. Bullougb, and M.R. Hayns // J. Nucl. Mater. 1976, v. 60, p. 246.

7. M. Griffiths // J. Nucl. Mater. 1988, v. 159, p. 190.

8. C.H. Woo // J. Nucl. Mater. 2000, v. 276, p. 90.

9. V.I. Dubinko, A.S. Abyzov, A.A. Turkin // J. Nucl. Mater. 2005, v. 336, p. 11.

10. C.H. Woo, U. Gosele // J. Nucl. Mater. 1983, v. 119 , p. 219.

11. G.D. Samolyuk, A.V. Barashev, S.I. Golubov, Y.N. Osetsky, R.E. Stoller // Acta Materialia. 2014, v. 78, p. 173 .

12. J.D. Eshelby // Solid State Physics. 1956, v. 3, p 79-144.

13. E. Kröner // Zeitschrift fur Phyzik. 1953, v. 136, p. 402 .

14. M.H. Yoo // Phys. Stat. Sol. (b). 1974, v. 61, p. 411.

15. L.D. Landau, E.M. Lifshitz. Theory of Elasticity. Butterworth-Heinemann, Oxford, 1986, v. 7.

16. O.G. Trotsenko, P.N. Ostapchuk // Problems of Atomic Science and Technology. 2017, N 2(108), p. 83.

17. L. Fast, J.M. Wills, B. Johansson, O. Eriksson // Phys. Rev. B. 1995, v. 51, p. 17431.

18. A.V. Babich, P.N. Ostapchuk // Problems of Atomic Science and Technology. 2019, N 5(123), p. 11. 


\section{ФАКТОР ПРЕДПОЧТЕНИЯ БАЗИСНОЙ ДИСЛОКАЦИОННОЙ ПЕТЛИ В ЦИРКОНИИ}

\section{А.В. Бабич, П.Н. Остапчук}

С помощью метода функции Грина для гексагональных кристаллов в подходе Кренера получено аналитическое выражение для энергии упругого взаимодействия радиационных точечных дефектов дипольного типа с базисной дислокационной петлей ГПУ-металла. Оно было использовано для численного расчета фактора предпочтения базисной дислокационной петли в цирконии в тороидальном резервуаре. Тороидальная геометрия резервуара позволяет провести расчеты для петли любого размера и без какой-либо коррекции упругого поля в ее области влияния. Получены зависимости фактора предпочтения петли от ее радиуса и природы при различной форме диполей точечных дефектов.

\section{ФАКТОР ПЕРЕВАГИ БАЗИСНОЇ ДИСЛОКАЦІЙНОЇ ПЕТЛІ В ЦИРКОНІЇ}

\section{А.В. Бабіч, П.М. Остапчук}

За допомогою методу функції Гріна для гексагональних кристалів у підході Кренера отримано аналітичний вираз для енергії пружної взаємодії радіаційних точкових дефектів дипольного типу з базисною дислокаційною петлею ГЩУ-металу. Його було використано для чисельного розрахунку фактора переваги базисної дислокаційної петлі в цирконії в тороїдальному резервуарі. Тороїдальна геометрія резервуару дозволяє проводити розрахунки для петлі будь-якого розміру і без будь-якої корекції пружного поля в області іiі впливу. Отримано залежності фактора переваги петлі від іï радіуса і природи для різної форми диполів точкових дефектів. 